\title{
Câncer do Colo do Útero no Brasil: Estado da Arte
}

A história do controle do câncer do colo do útero (CCU) começou a ser escrita há pouco mais de 100 anos. As três primeiras décadas do século passado concentraram grandes descobertas para essa área. Entre 1899 e 1911, Schauta e Wertein demonstraram a curabilidade do CCU por meio da cirurgia (histerectomia radical) ${ }^{1}$. Praticamente ao mesmo tempo, Dominici (1911) descobriu o princípio da infiltração do radium, que mais tarde seria utilizado com sucesso para a cura do câncer ${ }^{1}$, o que fez com que, a partir de 1920, se iniciasse um período marcado pelo afastamento gradual da cirurgia em favor da radioterapia ${ }^{2}$. Nessa mesma época, em 1925, Hinselmann inovou ao inspecionar o colo uterino com uma luneta, o que se traduziu no advento da colposcopia ${ }^{1}$. Pouco depois, em 1928, Papanicolaou ${ }^{3}$ e Babes ${ }^{4}$ apresentaram, à comunidade científica, a descrição inicial das alteraçôes citopatológicas do colo do útero. Mas a citologia como ferramenta diagnóstica somente seria introduzida em 1941, por Papanicolaou e Traut ${ }^{5}$, depois de superada a indiferença inicial ao método.

Não tardou para que as novidades chegassem ao Brasil. Em 1934, Arnaldo de Moraes, ao tomar conhecimento da colposcopia, torna-se o primeiro a utilizá-la no país. Apenas um ano após o lançamento da monografia de Papanicolaou e Traut ${ }^{5}$, a citologia esfoliativa foi defendida como método para o diagnóstico do CCU por Antonio Vespasiano Ramos, assistente do Dr. Moraes na Universidade do Brasil (atual Universidade Federal do Rio de Janeiro), em tese de docência intitulada "Novo método de diagnóstico precoce do câncer uterino"6. Há ainda registro de que Arnaldo de Moraes tenha sido o primeiro no mundo a utilizar, simultaneamente, colposcopia e citopatologia ${ }^{7}$. Apesar disso, o método era pouco reconhecido internacionalmente, a ponto de, em 1952, Ralston Paterson, uma das principais autoridades mundiais no tratamento do câncer por radioterapia, em artigo publicado na Revista Brasileira de Cancerologia (RBC) $)^{8}$, assumir sua pequena experiência com a técnica desenvolvida por Papanicolaou, comentar sobre o surgimento de "clínicas preventivas" em outros países, e destacar, equivocadamente, que, "uma vez que os cânceres do colo do útero desenvolvem-se muito rapidamente, a melhor prevenção era a terapêutica imediata... e não exames periódicos com intervalos de um ou dois anos".

Em 1956, foi fundado o primeiro Serviço de Citopatologia no Brasil, na Santa Casa de Belo Horizonte e, nesse mesmo ano, criada a Sociedade Brasileira de Citologia9. A apropriação dos conhecimentos fez com que, já em 1957, em artigo também publicado na RBC ${ }^{1}$, o Prof. Alberto Henrique Rocha alertasse a comunidade científica que "entre 30 e 50 anos, fase de maior incidência do câncer do colo do útero, toda mulher deveria submeter-se, pelo menos uma vez por ano, a um exame ginecológico, visando a descobrir o câncer inicial do colo uterino”. Começava aí uma mudança de paradigma, por meio do qual, além da cirurgia e da radioterapia, importantes para o tratamento de pacientes com CCU, valorizava-se sua deteç̧ão precoce, incentivando-se aquela que se tornaria uma das mais efetivas estratégias de prevenção secundária conhecida na atualidade: o rastreamento do CCU pelo exame de Papanicolaou. Nesse mesmo ano (1956), o então presidente Juscelino Kubitschek patrocinou a construção do Centro de Pesquisas Luíza Gomes de Lemos, da Fundação das Pioneiras Sociais, no Rio de Janeiro (atualmente Hospital do Câncer III / INCA) para atender aos casos de câncer da mama e do aparelho genital feminino. Acredita-se que essa tenha sido a primeira iniciativa de dimensão institucional direcionada para o controle do CCU em nosso país ${ }^{10}$. Foi um biólogo da Fundação das Pioneiras Sociais, Sérgio Ré de Paiva, que, em 1973, concebeu o uso de uma escovinha - que viria a ser mais tarde conhecida como escova de Campos da Paz - para a coleta de material de endocérvice. Atendendo a uma solicitação do seu professor, o Dr. Artur Campos da Paz, Paiva idealizou o instrumento após observar sua esposa passar uma escovinha de rímel nos cílios. De domínio público, atualmente, a escovinha é usada universalmente ${ }^{11}$. Neste número da RBC, um artigo de cunho histórico, de autoria de Temperini, destaca o pioneirismo e o caráter inovador da atuação da Fundação das Pioneiras Sociais na utilização do teste de Papanicolaou como estratégia de prevenção em massa.

As primeiras respostas estruturadas de caráter nacional do Governo Federal surgiram somente em 1980, quando o Ministério da Saúde lançou, em parceria com a Organização Pan-americana da Saúde (OPAS), um primeiro "Manual de normas e procedimentos para o controle do câncer cérvico-uterino". Pouco depois (1983/1984), a Divisão Nacional de Saúde Materno Infantil do Ministério da Saúde definiu as bases de Assistência Integral à Saúde da Mulher, incluindo, entre as recomendaçóes, açóes voltadas para o controle do CCU e da mama ${ }^{12}$.

No que diz respeito à população-alvo para a realização de exames preventivos para a detecção do CCU e sua frequência, segundo Faerstein ${ }^{13}$, o manual lançado em 1980 propunha que as açôes deveriam estar integradas às demais atividades de saúde pública; o exame deveria ser colhido com frequência anual, concentrando-se a atenção nas populaçóes de alto risco: mulheres de 20 a 49 anos, com início da atividade sexual precoce, multiparidade, gestação em 
idade precoce e baixo nível socioeconômico. Em 1986, uma atualização do manual propôs que, em caso de duas ou mais citologias negativas, os controles poderiam ser espaçados para 2 ou 3 anos, o que já tinha vinha sendo proposto, desde 1972, pela OPAS, por meio da Publicação Científica número 248: Manual de normas y procedimientos para el control del cancer del cuello uterino. Mas foi em 1988 que, após reunião de consenso, o Ministério da Saúde, com o apoio das sociedades científicas afins, propôs que, em mulheres de 25 a 60 anos, o exame preventivo do CCU fosse realizado a cada três anos após a obtençâo de dois resultados negativos com intervalo de um ano ${ }^{14}$. As recomendaçôes permanecem quase intocadas nos dias de hoje: indica-se o rastreamento para mulheres de 25 a 64 anos com intervalo entre os exames de três anos, após dois exames negativos com intervalo anual ${ }^{10}$.

A essas primeiras açóes somou-se, em 1986, a proposta de desenvolvimento de açôes descentralizadas nas áreas de prevenção do câncer do colo uterino por meio da Campanha Nacional de Combate ao Câncer / Ministério da Saúde ${ }^{15}$. Entretanto, no ano seguinte (1987), ao perceber que, "por falta de uma estrutura executiva mais ampla, as finalidades básicas da Campanha Nacional de Combate ao Câncer vinham sendo realizadas de maneira limitada no âmbito nacional", foi criado o Programa de Oncologia (PRO-ONCO), que estabeleceu, entre outros projetos, a expansão da prevenção e controle do CCU no país ${ }^{16}$. Em 1990, a Lei Orgânica da Saúde ${ }^{17}$ definiu o Instituto Nacional de Câncer (INCA) e a Fundação das Pioneiras Sociais como referenciais para o Sistema único de Saúde (SUS) na prestação de serviços, formação de recursos humanos e transferência de tecnologia, muito embora, desde os anos 1960, o INCA já desenvolvesse iniciativas de caráter nacional nos campos assistencial, científico e educacional.

Apesar do conhecimento científico disponível, dos investimentos governamentais e das normalizaçóes vigentes, o país conviveu por muitos anos com taxas de mortalidade por CCU inaceitáveis, carecendo de açóes estruturadas, abrangentes e contínuas para corrigi-las. Foi nesse cenário que o governo brasileiro assumiu, em 1995, durante a VI Conferência Mundial sobre a Mulher, ocorrida na China, o compromisso de desenvolver um programa visando ao controle do CCU no País. Como resposta, em 1996, o INCA lançou um projeto-piloto, denominado "Programa Viva Mulher", em cinco capitais e em um Estado, tendo como meta reduzir em 50\% a incidência do CCU no país. Dois anos mais tarde, com base na experiência do INCA, o Ministério da Saúde instituiu o Programa Nacional de Combate ao Câncer de Colo Uterino (PNCCCU) ${ }^{18}$ e lançou uma campanha nacional (18 de agosto a 30 de setembro de 1998) que atingiu todos os Estados e $98 \%$ dos municípios do Brasil. Pela primeira vez no país, a detecção precoce do CCU foi abordada de maneira uniforme seguindo as mesmas recomendaçóes técnicas do Ministério da Saúde. Em 2002, uma segunda campanha, de proporçôes ainda maiores, foi realizada nos meses de março e abril. Essas iniciativas são destacadas no artigo de Freitas, Silva e Thuler.

Os anos seguintes trouxeram o reconhecimento do câncer como um problema de saúde pública e o fortalecimento do INCA como executor, normalizador e coordenador da política nacional de controle do câncer no Brasil. O Ministério da Saúde publicou, em 2005, a Política Nacional de Atenção Oncológica, que incluiu entre seus componentes fundamentais o controle do CCU . Dada a importância do problema, em 2010, a presidente da república lançou o "Programa de Fortalecimento da Rede de Prevençấo, Diagnóstico e Tratamento do Câncer de Colo do Útero e de Mama".

Embora, em 1983, a equipe de zur Hausen tivesse estabelecido o HPV 16 como principal elemento na patogênese das neoplasias cervicais invasoras e pré-invasoras, foi somente em 2002 que, em editorial do New England Journal of Medicine ${ }^{20}$, Christopher Crum anunciou os primeiros resultados do ensaio clínico sobre a vacina contra HPV questionando se esse seria o "início do fim do câncer cervical". Desde então, muitos avanços têm sido registrados no diagnóstico e na prevenção da infecção pelo HPV. Em um significativo artigo de opiniâo, Corrêa e Russomano destacam o inegável sucesso do rastreamento citológico de lesôes precursoras do CCU, e argumentam porque o modelo de prevenção, que vem sendo adotado nos últimos anos nos países desenvolvidos, baseado nos testes de detecção de HPV e na utilização da vacina contra HPV, não deve ser generalizado e importado sem a avaliação prévia da sua viabilidade, sustentabilidade e relação custo-efetividade e apresentam ao leitor as bases para as recomendaçôes atuais.

Um dos principais objetivos do PNCCCU, nos últimos anos, tem sido o aumento da cobertura do exame de Papanicolaou. O artigo de Murata, Gabrielloni e Schirmer mostra que, em Maringá, no Paraná, a cobertura do exame de Papanicolaou nos três anos anteriores foi de 87,6\% nas mulheres entre 25 e 59 anos, e que náo foi observada qualquer associação significativa entre os dados sociodemográficos e a submissão ao exame preventivo. Em outro artigo, Freitas, Silva e Thuler analisaram dados secundários do Estado de Mato Grosso do Sul e obtiveram, para 2003 e 2008, resultados ligeiramente inferiores de cobertura: $82,0 \%$ e $82,9 \%$, respectivamente.

Além de garantir cobertura, há uma preocupação crescente no país com a qualidade e desempenho dos exames, o que foi expresso em diversos artigos deste número. Bortolon e colaboradores, após avaliar a qualidade dos laboratórios de citopatologia do colo do útero no Brasil, destacaram, entre outros aspectos, a baixa capacidade da rede laboratorial para a identificaçáo de lesôes intraepiteliais de alto grau e a necessidade de programas de controle de qualidade do exame citopatológico. Bastos e colaboradores, por sua vez, a partir dos dados do SISCOLO do Estado do Rio de 
Janeiro, concluíram que a presença de elementos celulares representativos da zona de transformação do colo do útero na lâmina coletada para o exame citopatológico aumentou em cinco vezes a chance de detecção das atipias celulares. Por outro lado, Etlinger e colaboradores analisaram as discordâncias diagnósticas dos exames citopatológicos do programa de monitoramento externo de qualidade no Estado de São Paulo, entre 2000 e 2010, e estabeleceram que houve discordância diagnóstica em 16.581 (13,48\%) das 123.002 amostras revisadas, embora a concordância entre os diagnósticos originais e de revisão tenha sido considerada boa (índice Kappa=0,77). Ainda sobre esse assunto, Collaço e colaboradores apresentaram suas conclusóes sobre o controle de qualidade das biópsias e produtos de cirurgia de alta frequência no Programa de Prevenção do CCU do Estado do Paraná. As discrepâncias diagnósticas mais comuns foram entre lesão intraepitelial de baixo grau e diagnóstico de lesôes benignas (supradiagnóstico) e entre lesão intraepitelial de baixo grau e lesão intraepitelial de alto grau (subdiagnóstico). Foram encontradas taxas de concordância excelentes (índice Kappa= 0,99). Por seu turno, Fernandes e colaboradores, no Rio de Janeiro, estudaram as células escamosas atípicas de significado indeterminado no Serviço Integrado Tecnológico em Citologia (SITEC) / INCA e detectaram que sua frequência ficou abaixo de 5\%. ASC-US foi mais diagnosticado em faixas etárias mais jovens, enquanto ASC-H sofreu pouca variação entre as diferentes faixas etárias. Em outro estudo, Prado e colaboradores caracterizaram o perfil das mulheres com alteraçôes citológicas em Rio Branco, no Acre, e concluíram pela necessidade de melhorias no programa de prevenção do CCU no Estado. O estudo mostrou que 54,0\% das mulheres com resultados colpocitológicos de ASCUS/AGC, LSIL e HSIL não receberam tratamento após o exame alterado e que $45,7 \%$ dos diagnósticos citológicos de atipias de significado indeterminado revelaram-se lesóes de alto grau ou câncer no histopatológico. Solé Pla e colaboradores compararam o perfil dos exames citopatológicos do colo do útero em mulheres indígenas àquele das mulheres não indígenas no país, e revelaram que a razão entre lesão de alto grau / câncer invasor do colo do útero passou de 11,5, em 2009, para 16,1, em 2011, em não indígenas e de 1,7 para 5,0, entre indígenas, respectivamente.

Ao lado da preocupaçáo com o desempenho do exame, estão os esforços para se ter um sistema de informação fidedigno. A criação de um aplicativo para a informatização dos resultados dos exames citopatológicos, o Sistema de Informação do Câncer do Colo do Útero (SISCOLO), no final dos anos $1990^{22}$, representou importante avanço para o monitoramento e a avaliação das açôes no Brasil. Neste número da RBC, Almeida e colaboradores, ao avaliar a qualidade dos dados do SISCOLO de Vitória, concluíram por sua acessibilidade e oportunidade, apesar de problemas quanto à completude, principalmente dos dados epidemiológicos.

Estudos do perfil clinicoepidemiológico das pacientes com lesôes precursoras e CCU têm sido cada vez mais comuns no país. Silva e colaboradores apresentam a história natural das lesóes precursoras do CCU em uma coorte de 227 mulheres do Rio de Janeiro e apontam para maior propensão à regressão de lesões de baixo grau em mulheres com menos de 30 anos e de persistência ou progressão de lesôes de alto grau em mulheres com 50 anos ou mais. Em outro artigo, Thuler, Bergmann e Casado baseiam-se em dados de registros hospitalares de câncer de 77.317 casos de CCU diagnosticados no Brasil, entre 2000 e 2009, para apresentar o perfil demográfico e clínico das mulheres acometidas pela doença: jovens, de cor parda, com baixa escolaridade e com doença avançada ao diagnóstico. Já em Vitória, no Espírito Santo, Mascarello e colaboradores, em estudo que também teve como base registro hospitalar de câncer, apontaram que a idade, escolaridade, tipo histológico, recidiva e metástases estiveram associados ao estadiamento da doença. Em outro artigo, Vilaça e colaboradores analisaram as diferenças nos padróes de tratamento e nas características epidemiológicas entre pacientes adultas e idosas com CCU e observaram haver maior incidência de tumores avançados em mulheres idosas; também descreveram que, para esses mesmos estadiamentos, mulheres adultas foram submetidas mais frequentemente a procedimentos cirúrgicos, radioterapia e quimioterapia, enquanto nas idosas o tratamento mais comum foi a radioterapia isolada. Em uma tentativa de mudar esse cenário, por meio da proposição de novas abordagens à doença, Nogueira-Rodrigues e Melo, em artigo de opinião, discorreram sobre como as alteraçôes moleculares, que influenciam a fisiopatologia e o comportamento clínico do CCU, poderão se constituir em alvos terapêuticos no futuro, trazendo perspectivas promissoras no tratamento desse câncer.

A preocupação com as estratégias de enfrentamento da doença e a qualidade de vida das sobreviventes também tem sido alvo de atençâo. Panobianco e colaboradores, visando a compreender como mulheres com diagnóstico avançado do CCU enfrentam a doença e o tratamento, empreenderam um estudo qualitativo e concluíram pela importância das crenças individuais baseadas em experiências vividas anteriormente à doença. Por outro lado, a qualidade de vida de pacientes com CCU em tratamento radioterápico foi retratada no artigo de Santos e colaboradores. Após analisar mulheres com CCU tratadas com radioterapia adjuvante, exclusiva ou concomitante à quimioterapia, concluíram que a radioterapia não modificou a qualidade de vida relacionada à saúde dessas mulheres.

O papel do profissional de enfermagem na prevenção do CCU na atenção primária foi abordado em um estudo qualitativo apresentado por Melo e colaboradores, que evidenciaram a importância desse profissional, de sua integração 
com a equipe e a comunidade, do conhecimento da realidade local, do estabelecimento de vínculo e da avaliação constante dos resultados obtidos. Outro artigo tratou da interface do cuidado do CCU no complexo econômicoindustrial da saúde. Nele, Gomes, Lima e Kuschnir destacaram a dependência produtiva e tecnológica da expansão da atenção ao CCU e seu poder de alavancar a Estratégia Nacional de Inovação do Ministério da Ciência, Tecnologia e Inovação e o desenvolvimento industrial.

Diminuir a incidência e a mortalidade por CCU estão entre os objetivos atuais do PNCCCU ${ }^{20}$. No estudo apresentado por Freitas, Silva e Thuler, observa-se um incremento de 33,8\% nas taxas específicas de mortalidade por CCU, ajustadas por idade, no Estado de Mato Grosso do Sul, nos últimos 30 anos. Felizmente, essa realidade não se reproduz no país como um todo. Recentemente, ao corrigir as taxas de mortalidade entre 1981 e 2006, com base na redistribuição proporcional dos óbitos por câncer do útero "porção não especificada", Silva e colaboradores mostraram haver tendência decrescente nas taxas para o país como um todo, queda nas capitais em todas as regiôes; enquanto para os municípios do interior houve aumento nas Regiōes Norte e Nordeste, declínio nas Regiōes Sudeste e Sul e estabilidade no Centro-Oeste. Em artigo publicado neste número, Guimarães e colaboradores utilizam três diferentes técnicas estatísticas para mostrar o decrescimento dessas taxas no Brasil entre 1980 e 2009. Segundo os autores, a redução pode estar associada às açôes de rastreamento das lesóes precursoras do CCU e ao controle da transmissão do HPV.

Ousamos qualificar este editorial de "estado da arte" uma vez que ele sintetiza a produção acadêmica sobre CCU, das mais diferentes áreas do conhecimento, mapeada neste número temático da RBC. Ao concluí-lo, destacamos a importância da iniciativa de reunir essa multiplicidade de perspectivas e pluralidade de enfoques, permitindo o ordenamento do conjunto de informaçôes e de resultados obtidos por pesquisadores brasileiros. Sua leitura não deve ser linear ou em uma simples cadeia. Cada texto, mais do que ligado àqueles que o antecedem e o sucedem, traz, em seu interior, importantes enunciados. A imagem que melhor pode sintetizar essa compilação é a de "rede" e não de cadeia. Rede de fios que se unem, que se rompem, que se cruzam, que produzem...

Agradeçamos!

Boa leitura.

\section{Luiz Claudio Santos Thuler}

Tecnologista e Docente do Programa de Pós-Graduação em Oncologia

Instituto Nacional de Câncer José Alencar Gomes da Silva (INCA)

Professor Associado da Universidade Federal do Estado do Rio de Janeiro (UNIRIO)

Rio de Janeiro - Brasil 


\section{REFERÊNCIAS}

1. Rocha AL. Conceito atual do diagnóstico e tratamento do câncer incipiente do colo uterino: breve histórico do diagnóstico precoce do câncer do colo uterino. Rev bras cancerol. 1957;14(16):5-57.

2. Fried C. Tratamento do carcinoma do colo do útero: ensaio crítico dos métodos usados nos últimos 10 anos. Rev bras cancerol. 1951;4(7):6-30.

3. Classes in oncology: George Nicholas Papanicolaou's new cancer diagnosis presented at the Third Race Betterment Conference, Battle Creek, Michigan, January 2-6, 1928, and published in the Proceedings of the Conference. CA Cancer J Clin. 1973;23(3):174-9.

4. Babes A. Diagnostic du cancer du col utérin par les frottis. Presse Medicale. 1928;36: 451-4.

5. Papanicolaou GN, Traut HF. The diagnostic value of vaginal smears in carcinoma of the uterus. Am J Obstet Gynecol. 1941;42(2):193-205.

6. Eraso Y. Migrating techniques, multiplying diagnoses: the contribution of Argentina and Brazil to early 'detection policy' in cervical cancer. Hist ciênc saúde-Manguinhos. 2010;17(supl 1):33-51.

7. Tuoto EA. Biografias médicas: Arnaldo de Moraes [Internet]. [citado 2012 jul 27]. Disponível em: http://medbiography. blogspot.com.br/2006/06/arnaldo-de-moraes-incl-foto-photo.html

8. Paterson R. Câncer do colo do útero. Rev bras cancerol. 1952;6(9):5-16.

9. Xavier FE. História da citopatologia no Brasil. Informativo Citopatologia [Internet]. 2002 abr-jun [citado 2005 jan 25];(59). Disponível em: http://www.citopatologia.org.br

10. Instituto Nacional de Câncer (Brasil). Diretrizes brasileiras para o rastreamento do câncer do colo do útero. Rio de Janeiro: INCA; 2011. $104 \mathrm{p}$.

11. Instituto Nacional de Câncer (Brasil). Escovinha de maquiagem que salva vidas. Informe INCA. 2001;(94):2.

12. Abreu E, Lopes ER. Projeto de expansão da prevenção e controle do câncer cérvico-uterino. Rev bras cancerol. 1988;34(4):209-19.

13. Faerstein E. População alvo e frequência da deteç̧ão do câncer de colo uterino. Rev bras cancerol. 1989;35(1/2):19-22.

14. Instituto Nacional de Câncer (Brasil). Consenso - Periodicidade e faixa etária no exame de prevenção do câncer cérvicouterino. Rev bras cancerol. 1988;35(1/2):77.

15. Kligerman J. Fundamentos para uma política nacional de prevenção e controle do câncer. Rev bras cancerol. 2002;48(1):3-7.

16. Lopes ER, Abreu E, Rezende MC, Lavor MF. Prevenção e controle do câncer no Brasil: ações do Pró-Onco/INCa. Rev bras cancerol. 1994;40(2):105-10.

17. Brasil. Presidência da República. Casa Civil. Subchefia para Assuntos Jurídicos. Lei n. 8.080, de 19 de setembro de 1990. Dispõe sobre as condiçōes para a promoção, proteção e recuperação da saúde, a organização e o funcionamento dos serviços correspondentes e dá outras providências. Diário Oficial da União, Brasília (1990 set 20);Sec.1:1.

18. Brasil. Ministério da Saúde. Gabinete do Ministro. Portaria n. 3.040, de 21 de junho de 1998. Institui o Programa Nacional de Combate ao Câncer de Colo Uterino. Diário Oficial da União, Brasília (1998 jun 23);Sec.1:102.

19. Brasil. Ministério da Saúde. Portaria n. 2.439, de 8 de dezembro de 2005. Institui a Política Nacional de Atenção Oncológica: Promoção, Prevenção, Diagnóstico, Tratamento, Reabilitação e Cuidados Paliativos, a ser implantada em todas as unidades federadas, respeitadas as competências das três esferas de gestão. Diário Oficial da União, Brasília (2005 dez 9);Sec.1:80-1.

20. Crum CP. The beginning of the end for cervical cancer? N Engl J Med. 2002;347(21):1703-5.

21. Instituto Nacional de Câncer (Brasil). Programa Nacional de Controle do Câncer do Colo do Utero [Internet]. [citado 2012 julho 16]. Disponível em: http://www2.inca.gov.br/wps/wcm/connect/acoes_programas/site+/home/nobrasil/ programa_nacional_controle_cancer_colo_utero/

22. Brasil. Ministério da Saúde. Secretaria de Assistência à Saúde. Portaria n. 408 de 30 de julho de 1999. Determina que o pagamento dos procedimentos referentes à Citopatologia, Histopatologia, e controle de qualidade ficará vinculado à prestação de informações necessárias ao monitoramento e avaliação das atividades de controle do câncer de colo de útero no Brasil, por intermédio de BPA em meio magnético, gerado exclusivamente pelo sistema definido nesta portaria. Diário Oficial da União, Brasília (1999 ago 2);Sec.1:14.

23. Azevedo e Silva G, Girianelli VR, Gamarra CJ, Bustamante-Teixeira MT. Cervical cancer mortality trends in Brazil, 1981-2006. Cad Saúde Pública. 2010;26(12):2399-407. 